\title{
Axiomatik der metrischen Spintensoren in der projektiven Relativitätstheorie
}

\author{
Von ERnst Schmutzer \\ Aus dem Theoretisch-Physikalischen Institut der Universität Jena \\ (Z. Naturforschg. 15 a, 831-835 [1960] ; eingegangen am 30. Juni 1960)
}

\begin{abstract}
Auf der Grundlage zweier Axiome für die metrischen Spintensoren (Verallgemeinerung der PauliMatrizen) im projektiven, 5-dimensionalen gekrümmten Raum werden die verschiedenen algebraischen Relationen zwischen diesen Spintensoren hergeleitet. Dabei zeigt es sich, daß die zugehörigen Projek. tionen in die Raum-Zeit-Welt auf die für diese früher deduzierten Verknüpfungen führen. Die Kenntnis dieser Relationen ist wichtig für die logische Vereinigung von Spinortheorie und projektiver Relativitätstheorie.
\end{abstract}

Da die verallgemeinerte projektive Relativitätstheorie eine auf einer tensoriellen Geometrie basierende Feldtheorie ist, die die Erscheinungskomplexe: Gravitation, Elektromagnetismus und einen weiteren, in der physikalischen Deutung jedoch noch umstrittenen Komplex ${ }^{1}$ umfaßt, liegt der Wunsch nahe, einen konsistenten Einbau der Spinoren in diesen projektiv-relativistischen Rahmen zu erreichen, um auch quantenphysikalische Konsequenzen ziehen zu können. Hinsichtlich der Erforschung der Bispinoren liegen Untersuchungen von Ludwig ${ }^{2}$ und Rumer ${ }^{3}$ vor. Wie in einer kürzlich erschienenen Arbeit bemerkt ${ }^{4}$, ziehen wir aus gewissen Gründen die eigentliche Spinortheorie vor, die wir im Anschluß an INFELD und VAN DER WAERDEN in einigen Richtungen weiterentwickelt haben. Für die Fortführung dieser Gedanken auf der Basis der projektiv-relativistischen Theorie ist die Kenntnis der algebraischen Relationen der metrischen Spintensoren von entscheidender Bedeutung. Wir gehen dabei so vor, daß wir im Sinne von Harish-Chandra ${ }^{5}$ auf der Grundlage zweier Axiome den 4-dimensionalen Formelappparat so für den 5-dimensionalen projektiv-relativistischen Raum verallgemeinern, daß ersterer durch Radialund Flächenprojektion (Entwicklung dieses Projektionsapparates in früheren Arbeiten des Verf. ${ }^{1}$ ) aus dem verallgemeinerten Formelapparat eindeutig hervorgeht.

Wir wiederholen kurz die wichtigsten von uns früher gebrauchten Begriffe und Bezeichnungen, an die wir uns im wesentlichen wieder halten wollen: Kleine griechische Indizes beziehen sich auf den

1 E. Schmutzer, Z. Phys. 149, 329 [1957] ; 154, 312 [1959]; Wiss. Z. Univ. Jena, Jahrg. 8, 15 [1958/59], math.-naturw. Reihe (Zusammenfassung).

2 G. Ludwig, Fortschritte der projektiven Relativitätstheorie, Verlag Vieweg, Braunschweig 1951. projektiv-relativistischen Raum und laufen von $1-5$, während kleine lateinische Indizes der Raum-Zeit entsprechen und deshalb von $1-4$ zu nehmen sind. Beide Sorten von Indizes sind Tensorindizes. Große lateinische Indizes sind Spinorindizes und laufen von $1-2$ über die Dimensionen des komplexen Spinorraumes. $\left\{x^{i}\right\}$ sind die 4 Raum-Zeit-Koordinaten. Die zugehörige metrische Signatur sei +++- , so daß $\stackrel{4}{g}=-\left|g_{i j}\right|>0$ wird. $\left\{X^{\mu}\right\}$ sind die 5 projektiven Koordinaten, wobei wir $g=-\left|g_{\mu \nu}\right|>0$ wählen wollen. $\left\{\mathfrak{e}_{i}\right\}$ stellt das vektorielle 4 -Bein dar, das die Raum-Zeit-Welt aufspannt, während $\left\{\mathfrak{e}_{\mu}\right\}$ als vektorielles 5-Bein den projektiv-relativistischen Raum aufspannt. Letzterer kann auch durch die Vektoren $\left\{\mathfrak{e}_{i}, \Re=\mathfrak{e}_{\mu} X^{\mu}\right\}$ aufgespannt werden, wobei dann folgende Beziehungen gelten:

$$
\begin{aligned}
\mathrm{e}^{i} & =g_{\mu}^{i} \mathrm{e}^{\mu}, \quad \mathrm{e}^{v}=g_{i}^{v} \mathrm{e}^{i}+\frac{X^{\nu} \Re}{S^{2}}, \\
\mathfrak{e}_{i} \Re & =X_{i}=0, \quad g_{\mu}^{i}=\frac{\partial x^{i}}{\partial X^{\mu}}, \\
g_{i}^{v} g_{\mu}^{i} & =g_{\mu}^{v}-\frac{X^{v} X_{\mu}}{S^{2}}, \quad S^{2}=\Re^{2}=X_{\mu} X^{\mu} .
\end{aligned}
$$

Mit dem antisymmetrischen Spintensor $\gamma_{\mathrm{AB}}$, für den die Relation:

$$
\gamma_{\mathrm{AB}} \gamma^{\mathrm{CB}}=\gamma_{\mathrm{A}}{ }^{\mathrm{C}}=\delta_{\mathrm{A}}{ }^{\mathrm{C}} \quad(\text { KroneCKeR-Symbol })
$$

besteht, werden Spinorindizes folgendermaßen bewegt:

$$
a_{\mathrm{A}}=\gamma_{\mathrm{BA}} a^{\mathrm{B}}, \quad a^{\mathrm{A}}=\gamma^{\mathrm{AB}} a_{\mathrm{B}} .
$$

Der durch die spinoriellen Basisvektoren $\left\{\mathfrak{e}_{\dot{\mathrm{AB}}}\right\}$ aufgespannte, der Raum-Zeit-Welt zugeordnete Spinor-

3 J. B. Rumer, Untersuchungen zur 5-Optik, Moskau 1956 (russ.).

4 E. Schmutzer, Z. Naturforschg. 15 a, 355 [1960].

5 Harish-Chandra, Proc. Ind. Acad. Sci. 23, 152 [1946]. 
raum ist mit den Basisvektoren der letzteren folgendermaßen verknüpft:

$$
\mathrm{e}_{\dot{\mathrm{AB}}}=-\sigma_{i \dot{\mathrm{AB}}} \mathrm{e}^{i}, \quad \mathrm{e}_{i}=\frac{1}{2} \sigma_{i}^{\dot{\mathrm{AB}}} \mathrm{e}_{\dot{\mathrm{AB}} \mathrm{B}},
$$

wobei $\mathfrak{e}_{\dot{\mathrm{A}} \mathrm{B}}=\mathfrak{e}_{\mathrm{B} \dot{\mathrm{A}}}$ gilt. Die metrischen Spintensoren $\sigma_{i \dot{A B}}$ erscheinen damit als Skalarprodukte beider Arten von Basisvektoren.

\section{§ 1. Relationen zwischen den metrischen Spin- tensoren im projektiv-relativistischen Raum}

Wir definieren die metrischen Spintensoren $\sigma_{\mu \dot{\mathrm{A} B}}$ im projektiv-relativistischen Raum in Verallgemeinerung der oben zitierten Überlegungen als die Skalarprodukte:

$$
\sigma_{\mu \dot{\mathrm{AB}}}=-\mathfrak{e}_{\dot{\mathrm{AB}}} \mathrm{e}_{\mu}=\sigma_{i \dot{\mathrm{AB}}} \mathrm{e}^{i} \mathrm{e}_{\mu}=\sigma_{i \dot{\mathrm{AB}}} g_{\mu}^{i} .
$$

Durch Multiplikation mit $g_{j}{ }^{\mu}$ resultiert daraus:

$$
\sigma_{\mu \dot{\mathrm{AB}}} g_{j}^{\mu}=\sigma_{j \dot{\mathrm{AB}}},
$$

und durch Multiplikation mit $X^{\mu}$ gewinnt man:

$$
\sigma_{\mu \dot{\mathrm{AB}}} X^{\mu}=\sigma_{i \dot{\mathrm{AB}}} X^{i}=0 .
$$

Die algebraischen Relationen zwischen den metrischen Spintensoren werden nun durch die beiden folgenden Axiome festgelegt:

Axiom I : $\sigma_{\mu \dot{\mathrm{B}}}=\sigma_{\mu \mathrm{B} \dot{\mathrm{A}}}($ Hermitezität),

Axiom II: $\sigma_{\mu} \dot{\mathrm{B}}_{\mathrm{A}} \sigma_{\nu \dot{\mathrm{BC}}}=g_{\mu \nu} \gamma_{\mathrm{AC}}+A X_{\mu} X_{v} \gamma_{\mathrm{AC}}$

$$
+B \varepsilon_{\mu \nu \varrho \tau \alpha} \frac{X^{\alpha}}{S} \sigma^{\circ \dot{\mathrm{B}}}{ }_{\mathrm{A}} \sigma^{\tau} \dot{\mathrm{BC}} \text {. }
$$

Dieses letzte Axiom stellt die allgemeinste Zerlegung des $\sigma$-Produktes in metrische Größen nach symmetrischen und antisymmetrischen Anteilen dar. Dabei sind $A$ und $B$ zunächst noch beliebige Ansatzkonstanten, die aus der inneren Konsistenz des Axioms zu bestimmen sind. Man beachte schließlich noch die Invarianzkonsistenz des Axioms bei der Vertauschung $\mu \longleftrightarrow v, \mathrm{~A} \longleftrightarrow \mathrm{C}$. Zur Berechnung der Konstanten $A$ und $B$ sowie für andere spätere Rechnungen benötigen wir Ausdrücke für die verschiedenen Produkte zwischen den Levi-Civitaschen
Pseudotensoren:

$$
\varepsilon_{\mu \nu \alpha \beta \gamma}=\sqrt{g} \delta^{\mu \nu \alpha \beta \gamma}, \quad \varepsilon^{\mu v \alpha \beta \gamma}=-\frac{1}{\sqrt{g}} \delta^{\mu v \alpha \beta \gamma} .
$$

Bei Beachtung der Symmetrieeigenschaften und der entsprechenden Normierung gelangt man zu folgenden Darstellungen:

$$
\begin{aligned}
& \varepsilon_{\mu \nu \varrho \tau \alpha} \varepsilon^{\mu \sigma \lambda . \varkappa \beta} \\
& =g_{\nu}^{\sigma} g_{\varrho}^{\lambda} g_{\tau}^{\beta} g_{\alpha}^{\alpha}+g_{\nu}^{\sigma} g_{\varrho}^{\alpha} g_{\tau}^{\lambda} g_{\alpha}^{\beta}+g_{\nu}^{\sigma} g_{\varrho}^{\beta} g_{\tau}^{\chi} g_{\alpha}^{\lambda}+g_{\nu}^{\lambda} g_{\varrho}^{\sigma} g_{\tau}^{\alpha} g_{\alpha}^{\beta} \\
& +g_{v}^{\alpha} g_{0}^{\sigma} g_{\tau}^{\beta} g_{\alpha}^{\lambda}+g_{v}^{\beta} g_{0}^{\sigma} g_{\tau}^{\lambda} g_{\alpha}^{\alpha}+g_{v}^{\beta} g_{0}^{\alpha} g_{\tau}^{\sigma} g_{\alpha}^{\lambda}+g_{\nu}^{\alpha} g_{\varrho}^{\lambda} g_{\tau}^{\sigma} g_{\alpha}^{\beta} \\
& +g_{v}^{\lambda} g_{\varrho}^{\beta} g_{\tau}^{\sigma} g_{\alpha}^{\alpha}+g_{v}^{\alpha} g_{\varrho}^{\beta} g_{\tau}^{\lambda} g_{\alpha}^{\sigma}+g_{v}^{\beta} g_{\varrho}^{\lambda} g_{\tau}^{\alpha} g_{\alpha}^{\sigma}+g_{v}^{\lambda} g_{\varrho}^{\chi} g_{\tau}^{\beta} g_{\alpha}^{\sigma} \\
& -g_{\nu}^{\sigma} g_{\varrho}^{\lambda} g_{\tau}^{\chi} g_{\alpha}^{\beta}-g_{v}^{\sigma} g_{\alpha}^{\beta} g_{\tau}^{\lambda} g_{\alpha}^{\alpha}-g_{\nu}^{\sigma} g_{\varrho}^{\alpha} g_{\tau}^{\beta} g_{\alpha}^{\lambda}-g_{v}^{\lambda} g_{\varrho}^{\sigma} g_{\tau}^{\beta} g_{\alpha}^{\alpha} \\
& -g_{v}^{\beta} g_{0}^{\sigma} g_{\tau}^{\gamma} g_{\alpha}^{\lambda}-g_{v}^{\alpha} g_{0}^{\sigma} g_{\tau}^{\lambda} g_{\alpha}^{\beta}-g_{v}^{\alpha} g_{0}^{\beta} g_{\tau}^{\sigma} g_{\alpha}^{\lambda}-g_{v}^{\beta} g_{\alpha}^{\lambda} g_{\tau}^{\sigma} g_{\alpha}^{\alpha}
\end{aligned}
$$

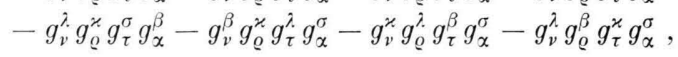

$$
\begin{aligned}
& \varepsilon_{\mu v \varrho \tau \alpha} \varepsilon^{u v \lambda, \beta \beta}=2\left[g_{\varrho}^{\lambda} g_{\tau}^{\beta} g_{\alpha}^{\alpha}+g_{0}^{\alpha} g_{\tau}^{\lambda} g_{\alpha}^{\beta}+g_{0}^{\beta} g_{\tau}^{\alpha} g_{\alpha}^{\lambda}\right. \\
& \left.-g_{\varrho}^{\lambda} g_{\tau}^{\alpha} g_{\alpha}^{\beta}-g_{\varrho}^{\beta} g_{\tau}^{\lambda} g_{\alpha}^{\alpha}-g_{\varrho}^{\alpha} g_{\tau}^{\beta} g_{\alpha}^{\lambda}\right] \text {, } \\
& \varepsilon_{\mu v \underline{Q} \tau \alpha} \varepsilon^{\mu v v_{Q} \times \beta}=6\left[g_{\tau}^{\beta} g_{\alpha}^{\alpha}-g_{\tau}^{\alpha} g_{\alpha}^{\beta}\right], \\
& \varepsilon_{\mu v \varrho \tau \alpha} \varepsilon^{\mu v v_{Q} \beta}=-24 g_{\alpha}^{\beta}, \\
& \varepsilon_{\mu v Q \tau \alpha} \varepsilon^{\mu v 0 \tau \alpha}=-5 \text { ! }
\end{aligned}
$$

Nach diesen Vorbereitungen überprüfen wir jetzt die Konsistenz von (9): Wir gehen aus diesem Grunde mit der linken Seite von (9) in die rechte Seite von (9) ein und erhalten bei Verwendung von (12):

$$
\begin{aligned}
\sigma_{\mu}{ }_{\mathrm{A}}^{\dot{\mathrm{B}}} \sigma_{\nu \dot{\mathrm{BC}}}=g_{\mu \nu} \gamma_{\mathrm{AC}} & +A X_{\mu} X_{\nu} \gamma_{\mathrm{AC}} \\
& -4 B^{3} \varepsilon_{\mu \nu \varrho \tau \alpha} \frac{X^{\alpha}}{S} \sigma^{\alpha \dot{\mathrm{B}}} \sigma^{\tau} \dot{\mathrm{BC}}{ }^{\mathrm{A}} .
\end{aligned}
$$

Daraus liest man dann sofort durch Vergleich mit (9) ab:

$$
B= \pm i / 2 .
$$

Das Auftreten beider Vorzeichen ist aus transformationstechnischen Gründen erforderlich.

Durch Multiplikation von (9) mit $X^{\mu}$ resultiert für die zweite Unbekannte $A$ :

$$
A=-1 / S^{2},
$$

so daß wir das Axiom II in der folgenden endgültigen Form schreiben können:

$$
\sigma_{\mu}^{\dot{\mathrm{B}}}{ }_{\mathrm{A}} \sigma_{\nu \dot{\mathrm{BC}}}=g_{\mu \nu} \gamma_{\mathrm{AC}}-\frac{X_{\mu} X_{v}}{S^{2}} \gamma_{\mathrm{AC}} \pm \frac{i}{2} \varepsilon_{\mu \nu \varrho \tau \alpha} \frac{X^{\alpha}}{S} \sigma^{o \dot{\mathrm{B}}}{ }_{\mathrm{A}} \sigma^{\tau} \dot{\mathrm{BC}} \cdot
$$

Durch Addition und Subtraktion mit vertauschten Indizes ergeben sich daraus die bemerkenswerten Vertauschungsregeln:

$$
\begin{aligned}
& \sigma_{\mu} \dot{\mathrm{B}}_{\mathrm{A}} \sigma_{\nu} \dot{\mathrm{BC}}+\sigma_{v}{ }_{\mathrm{A}}^{\dot{\mathrm{B}}} \sigma_{\mu \dot{\mathrm{BC}}}=2\left(g_{\mu \nu}-\frac{X_{\mu} X_{v}}{S^{2}}\right) \gamma_{\mathrm{AC}}, \\
& \sigma_{\mu}{ }_{\mathrm{A}}^{\dot{\mathrm{B}}} \sigma_{v \dot{\mathrm{BC}}}-\sigma_{\nu}{ }_{\mathrm{A}}^{\dot{\mathrm{B}}} \sigma_{\mu \dot{\mathrm{BC}}}= \pm i \varepsilon_{\mu v ? \tau \alpha} \frac{X_{S}^{\alpha}}{{ }^{\alpha}} \sigma^{2 \dot{\mathrm{B}}}{ }_{\mathrm{A}} \sigma^{\tau}{ }_{\dot{\mathrm{BC}}} \text {, }
\end{aligned}
$$


die wir als die richtigen Verallgemeinerungen der 4-dimensionalen Vertauschungsregeln anzusehen haben [vgl. Regel (20) in der Bispinorform nach LubwiG $^{2}$ !]. Durch Verjüngung gewinnt man daraus die weiteren Formeln:

$$
\begin{gathered}
\sigma_{\mu}^{\dot{\mathrm{BA}}} \sigma_{v \dot{\mathrm{BA}}}=-2\left(g_{\mu v}-\frac{X_{\mu} X_{v}}{S^{2}}\right), \\
\sigma^{\mu \dot{\mathrm{BA}}} \sigma_{\mu \dot{\mathrm{BA}}}=-8, \\
\sigma^{\mu \dot{\mathrm{B}}}{ }_{\mathrm{A}} \sigma_{\mu \dot{\mathrm{BC}}}=4 \gamma_{\mathrm{AC}} .
\end{gathered}
$$

Multipliziert man nun (19) mit $\varepsilon^{\mu \nu \lambda \varkappa \beta} \sigma_{\lambda \dot{\mathrm{D}}}{ }^{\mathrm{C}} X_{\beta}$ und verwendet man (12) zur Elimination des $\varepsilon$-Produktes, so findet man:

$4 \sigma_{\dot{\mathrm{DA}}}^{\alpha}=\sigma^{\alpha \dot{\mathrm{B}}}{ }_{\mathrm{C}} \sigma_{\dot{\mathrm{BA}}}^{\tau} \sigma_{\tau \dot{\mathrm{D}}}^{\mathrm{C}} \pm \mathrm{i} \varepsilon^{\mu \nu \lambda \varkappa \beta} \frac{X_{\beta}}{S} \sigma_{\mu}^{\dot{\mathrm{B}}} \sigma_{\nu \dot{\mathrm{B}}} \sigma_{\lambda \dot{\mathrm{D}}}^{\mathrm{C}}$.
Auf die ersten beiden Faktoren des ersten Gliedes der rechten Seite wenden wir abermals (19) an, so daß nach einigen Umformungen schließlich folgt:

$$
6 \sigma_{\dot{\mathrm{DA}}}^{\varkappa}= \pm \mathrm{i} \varepsilon^{\varkappa \mu \nu \lambda \beta} \frac{X_{\beta}}{S} \sigma_{\lambda \dot{\mathrm{D}}}{ }^{\mathrm{C}} \sigma_{\nu}{ }_{\mathrm{C}} \sigma_{\mu \dot{\mathrm{BA}}} .
$$

Eliminiert man die letzten beiden Faktoren der rechten Seite wiederum vermöge (19), so resultiert bei Benutzung von (12) :

$$
2 \sigma_{\dot{\mathrm{DA}}}^{\varkappa}=-\sigma_{\tau \dot{\mathrm{D}}}^{\mathrm{C}} \sigma_{\dot{\mathrm{B} A}}^{\tau} \sigma^{\alpha \dot{\mathrm{B}}}{ }_{\mathrm{C}}
$$

und daraus

$$
\sigma_{\mathrm{DC}}^{\tau} \sigma_{\tau \dot{\mathrm{BA}}}=2 \gamma_{\dot{\mathrm{DB}}} \gamma_{\mathrm{AC}},
$$

eine Formel, die für viele Umformungen von groBer Wichtigkeit ist.

Multipliziert man (19) mit $\sigma_{\dot{\mathbf{D}}}^{\lambda} \mathbf{C}$, so erhält man als Zwischenformel:

$$
\sigma_{\mu} \dot{\mathrm{B}} \sigma_{\nu \dot{\mathrm{BC}}} \sigma_{\dot{\mathrm{D}}}^{\lambda} \dot{\mathrm{C}}=-g_{\mu \nu} \sigma_{\dot{\mathrm{DA}}}^{\lambda}+\frac{X_{\mu} X_{v}}{S^{2}} \sigma_{\dot{\mathrm{DA}}}^{\lambda} \pm \frac{i}{2} \varepsilon_{\mu \nu \varrho \tau \alpha} \frac{X^{\alpha}}{S} \sigma^{\varrho \dot{\mathrm{B}}}{ }_{\mathrm{A}} \sigma_{\dot{\mathrm{D}}}^{\lambda} \sigma^{\mathrm{C}} \dot{\mathrm{BC}}
$$

Die letzten beiden Faktoren des letzten Gliedes der rechten Seite werden nun vermöge der aus (19) resultierenden Relation:

$$
\sigma_{\dot{\mathrm{D}}}^{\lambda} \sigma^{\tau} \dot{\mathrm{BC}}=g^{\lambda \tau} \gamma_{\dot{\mathrm{D}} \dot{\mathrm{B}}}-\frac{X^{\lambda} X^{\tau}}{S^{2}} \gamma_{\dot{\mathrm{D}} \dot{\mathrm{B}}} \mp \frac{i}{2} \varepsilon^{\lambda \tau \varepsilon \sigma \beta} \frac{X_{\beta}}{S} \sigma_{\varepsilon}^{\mathrm{C}} \dot{\mathrm{D}} \sigma_{\sigma \dot{\mathrm{BC}}}
$$

eliminiert, so daß entsteht:

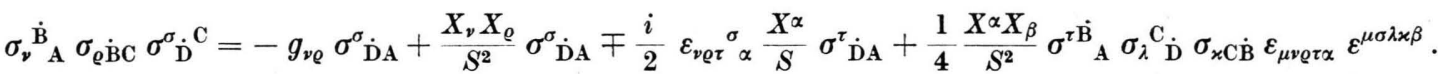

Auf das $\varepsilon$-Produkt wenden wir jetzt (11) an. Dann bekommen wir nach einer etwas längeren Zwischenrechnung:

$$
\begin{aligned}
& 4 \sigma_{\nu} \dot{\mathrm{B}} \sigma_{\varrho \dot{\mathrm{BC}}} \sigma_{\sigma \dot{\mathrm{D}}}{ }^{\mathrm{C}}=-4 g_{\nu \varrho} \sigma_{\sigma \dot{\mathrm{DA}}}+6 \sigma_{\varrho \dot{\mathrm{DA}}} g_{\sigma \nu}-6 \sigma_{\nu \dot{\mathrm{DA}}} g_{\varrho \sigma}+4 \frac{X_{\nu} X_{\varrho}}{S^{2}} \sigma_{\sigma \dot{\mathrm{DA}}}+6 \frac{X_{\sigma} X_{\varrho}}{S^{2}} \sigma_{\nu \dot{\mathrm{DA}}} \\
& -6 \frac{X_{\sigma} X_{v}}{S^{2}} \sigma_{\varrho \dot{\mathrm{DA}}} \mp 2 i \varepsilon_{\nu \varrho \tau \sigma \alpha} \frac{X^{\alpha}}{S} \sigma^{\tau} \dot{\mathrm{DA}}-\sigma_{\sigma} \dot{\mathrm{B}}_{\mathrm{A}}\left(\sigma_{\nu}^{\mathrm{C}} \dot{\mathrm{D}} \sigma_{\varrho \mathrm{CB}}-\sigma_{\varrho}{ }^{\mathrm{C}} \dot{\mathrm{D}} \sigma_{\nu} \dot{\mathrm{CB}}\right) \text {. }
\end{aligned}
$$

Für den Klammerausdruck des letzten Gliedes der rechten Seite benutzen wir die folgende Umformung:

$$
\sigma_{\nu}{ }^{\mathrm{C}} \dot{\mathrm{D}} \sigma_{\varrho \mathrm{C} \dot{\mathrm{B}}}-\sigma_{\varrho}{ }_{\varrho}^{\mathrm{C}} \dot{\mathrm{D}} \sigma_{\nu \mathrm{C} \dot{\mathrm{B}}}=2 \sigma_{\nu \dot{\mathrm{D}}}^{\mathrm{C}} \sigma_{\varrho \dot{\mathrm{BC}}}-2 g_{\varrho v} \gamma_{\dot{\mathrm{D}} \dot{\mathrm{B}}}+2 \frac{X_{\varrho} X_{v}}{S^{2}} \gamma_{\dot{\mathrm{D}} \dot{\mathrm{B}}},
$$

so daß schließlich folgt:

$$
\begin{aligned}
\sigma_{\nu}{ }_{\mathrm{A}}^{\dot{\mathrm{B}}} \sigma_{\varrho \dot{\mathrm{B} C}} \sigma_{\sigma \dot{\mathrm{D}}}{ }^{\nu} & +\sigma_{\varrho \dot{\mathrm{BC}}}\left(\sigma_{\nu \dot{\mathrm{D}}}{ }^{\mathrm{C}} \sigma_{\sigma}{ }_{\mathrm{A}}^{\dot{\mathrm{B}}}+\sigma_{\nu} \dot{\mathrm{B}}_{\mathrm{A}} \sigma_{\sigma \dot{\mathrm{D}}}{ }^{\mathrm{C}}\right)=-3 g_{\nu \varrho} \sigma_{\sigma \dot{\mathrm{DA}}}+3 g_{v \sigma} \sigma_{\varrho \dot{\mathrm{DA}}}-3 g_{\varrho \sigma} \sigma_{\nu \dot{\mathrm{DA}}} \\
& +3 \frac{X_{\nu} X_{\varrho}}{S^{2}} \sigma_{\sigma \dot{\mathrm{DA}}}+3 \frac{X_{\sigma} X_{\varrho}}{S^{2}} \sigma_{\nu \dot{\mathrm{DA}}}-3 \frac{X_{\sigma} X_{v}}{S^{2}} \sigma_{\varrho \dot{\mathrm{DA}}} \mp i \varepsilon_{\nu \varrho \tau \sigma \alpha} \frac{X^{\alpha}}{S} \sigma^{\tau} \dot{\mathrm{DA}} .
\end{aligned}
$$

Das zweite Glied der linken Seite soll noch weiter vereinfacht werden. Dazu sind aber noch einige Zwischenrechnungen nötig, die wir folgendermaßen durchführen wollen: Wir gehen aus von Beziehung (26), die wir in folgender Form schreiben wollen:

$$
6 \sigma^{\mu} \dot{\mathrm{DA}}= \pm i \varepsilon^{\mu \sigma \lambda \varkappa \beta} \frac{X_{\beta}}{S} \sigma_{\varkappa \dot{\mathrm{D}}}^{\mathrm{C}} \sigma_{\lambda}^{\dot{\mathrm{B}}}{ }_{\mathrm{C}} \sigma_{\sigma \dot{\mathrm{BA}}} .
$$


Nach Multiplikation mit $\varepsilon_{\mu \nu \text { ova }}$ und Elimination des $\varepsilon$-Produkts vermöge (11) ergibt sich ein sehr langer Ausdruck, auf dessen Niederschreiben wir verzichten wollen. Mit Hilfe von (20) läßt sich dieser Ausdruck auf die folgende angedeutete Struktur bringen:

$$
\begin{aligned}
& 6 \sigma_{\dot{\mathrm{D} A}}^{\mu} \varepsilon_{\mu \nu \rho \tau \alpha}= \pm \frac{2 i}{S}\left[X_{\tau}\left(\sigma_{\nu \dot{\mathrm{BA}}} \sigma_{\alpha \dot{\mathrm{D}}}^{\mathrm{C}} \sigma_{\varrho}^{\dot{\mathrm{B}} \mathrm{C}}+\sigma_{\varrho \dot{\mathrm{BA}}} \sigma_{\nu \dot{\mathrm{D}}}^{\mathrm{C}} \sigma_{\alpha}{ }_{\mathrm{C}}^{\dot{\mathrm{B}}}+\sigma_{\alpha \dot{\mathrm{BA}}} \sigma_{\varrho \dot{\mathrm{D}}}^{\mathrm{C}} \sigma_{\nu} \dot{\mathrm{B}} \mathrm{C}\right)+X_{\alpha}()+X_{\varrho}()+X_{v}()\right] \\
& \mp \frac{2 i}{S}\left[X_{\tau}\left(\sigma_{v \dot{\mathrm{DA}}} g_{\alpha \varrho}+\sigma_{\varrho \dot{\mathrm{DA}}} g_{v \alpha}+\sigma_{\alpha \dot{\mathrm{DA}}} g_{\varrho v}\right)+X_{\alpha}()+X_{\varrho}()+X_{v}(\quad)\right] \\
& \pm \frac{2 i}{S^{3}}\left[X_{\tau}\left(\sigma_{v \dot{\mathrm{DA}}} X_{\alpha} X_{\varrho}+\sigma_{\varrho \dot{\mathrm{DA}}} X_{v} X_{\alpha}+\sigma_{\alpha \dot{\mathrm{DA}}} X_{\varrho} X_{v}\right)+X_{\alpha}()+X_{\varrho}()+X_{v}(\quad)\right] \text {. }
\end{aligned}
$$

Multipliziert man diese Gleichung mit $X^{v}$, so fallen viele Glieder weg und wir gewinnen die Gleichung:

$$
\begin{aligned}
6 \sigma^{\mu} \dot{\mathrm{DA}} \varepsilon_{\mu \nu \varrho \tau \alpha} X^{v}= \pm 2 i S\left(\sigma_{\rho \dot{\mathrm{BA}}} \sigma_{\alpha \dot{\mathrm{D}}}^{\mathrm{C}} \sigma_{\tau}^{\dot{\mathrm{B}}}{ }_{\mathrm{C}}+\sigma_{\tau \dot{\mathrm{BA}}} \sigma_{\varrho \dot{\mathrm{D}}}^{\mathrm{C}} \sigma_{\alpha} \dot{\mathrm{B}}_{\mathrm{C}}+\sigma_{\alpha \dot{\mathrm{BA}}} \sigma_{\tau \dot{\mathrm{D}}}{ }^{\mathrm{C}} \sigma_{\varrho}{ }^{\dot{\mathrm{B}}} \mathrm{C}\right) \\
\mp 2 i S\left(\sigma_{\varrho \dot{\mathrm{DA}}} g_{\alpha \tau}+\sigma_{\tau \dot{\mathrm{DA}}} g_{\alpha \varrho}+\sigma_{\alpha \dot{\mathrm{DA}}} g_{\tau \varrho}\right) \pm \frac{2 i}{S}\left(X_{\varrho} X_{\alpha} \sigma_{\tau \dot{\mathrm{DA}}}+X_{\alpha} X_{\tau} \sigma_{\varrho \dot{\mathrm{DA}}}+X_{\tau} X_{\varrho} \sigma_{\alpha \dot{\mathrm{DA}}}\right) .
\end{aligned}
$$

Durch Symmetrisierung in $\alpha$ und $\tau$ und Verwendung von (20) resultiert schließlich daraus:

$$
\begin{aligned}
& \sigma_{\varrho \dot{\mathrm{BC}}}\left(\sigma_{\alpha{ }_{\mathrm{A}}}^{\dot{\mathrm{B}}} \sigma_{v \dot{\mathrm{D}}}+\sigma_{\nu} \dot{\mathrm{B}}{ }_{\alpha} \sigma_{\alpha \dot{\mathrm{D}}}{ }^{\mathrm{C}}\right) \\
& =\frac{2}{S^{2}}\left[\sigma_{\alpha \dot{\mathrm{DA}}} X_{\varrho} X_{v}+\sigma_{v \dot{\mathrm{DA}}} X_{\alpha} X_{\varrho}-\sigma_{\varrho \dot{\mathrm{DA}}} X_{\alpha} X_{v}\right]-2\left[\sigma_{v \dot{\mathrm{DA}}} g_{\alpha \varrho}+\sigma_{\alpha \dot{\mathrm{DA}}} g_{v \varrho}-\sigma_{\varrho \dot{\mathrm{DA}}} g_{\alpha v}\right]
\end{aligned}
$$

Damit können wir jetzt das entscheidende Glied von (31) eliminieren und erhalten dann für das Dreierprodukt:

$\sigma_{\nu}^{\dot{\mathrm{B}}}{ }_{\mathrm{A}} \sigma_{\varrho \dot{\mathrm{BC}}} \sigma_{\sigma \dot{\mathrm{D}}} \dot{\mathrm{C}}^{2} g_{v \sigma} \sigma_{\varrho \dot{\mathrm{DA}}}-g_{\varrho \sigma} \sigma_{v \dot{\mathrm{DA}}}-g_{v \varrho} \sigma_{\sigma \dot{\mathrm{DA}}}+\frac{X_{\sigma} X_{\varrho}}{S^{2}} \sigma_{v \dot{\mathrm{DA}}}+\frac{X_{v} X_{\varrho}}{S^{2}} \sigma_{\sigma \dot{\mathrm{DA}}}-\frac{X_{\sigma} X_{v}}{S^{2}} \sigma_{\varrho \dot{\mathrm{DA}}} \mp i \varepsilon_{v \varrho \tau \sigma \alpha} \frac{X^{\alpha}}{S} \sigma_{\dot{\mathrm{D} A}}^{\tau}$.

Für verschiedene Rechnungen benötigt man auch Viererprodukte. Deshalb multiplizieren wir die letzte Gleichung mit $\sigma_{\gamma}{ }_{F}$ durch. Die dann auftretenden Zweierprodukte eliminieren wir vermöge (19) und erhalten nach einigen Umformungen:

$$
\begin{aligned}
& \sigma_{\nu} \dot{\mathrm{B}}_{\mathrm{A}} \sigma_{\varrho \dot{\mathrm{BC}}} \sigma_{\sigma \dot{\mathrm{D}}}{ }^{\mathrm{C}} \sigma_{\gamma} \dot{\mathrm{D}}_{\mathrm{F}}=\gamma_{\mathrm{FA}}\left(g_{\nu \sigma} g_{\gamma \varrho}-g_{\varrho \sigma} g_{\gamma \nu}-g_{\nu \varrho} g_{\gamma \sigma}\right)+\frac{\gamma_{\mathrm{FA}}}{S^{2}}\left(g_{\varrho \sigma} X_{\gamma} X_{\nu}+g_{\nu \varrho} X_{\gamma} X_{\sigma}-g_{\nu \sigma} X_{\gamma} X_{\varrho}\right. \\
& \left.+g_{\gamma v} X_{\varrho} X_{\sigma}+g_{\gamma \sigma} X_{v} X_{\varrho}-g_{\gamma \varrho} X_{v} X_{\sigma}\right)-\frac{\gamma_{\mathrm{FA}}}{S^{4}} X_{v} X_{\sigma} X_{\gamma} X_{\varrho} \pm \frac{i}{2} \frac{X^{\alpha}}{S} \sigma^{\alpha \dot{\mathrm{B}}} \sigma^{\tau} \dot{\mathrm{BA}}\left(g_{v \sigma} \varepsilon_{\gamma \varrho x \tau \alpha}-g_{\varrho \sigma} \varepsilon_{\gamma v \kappa \tau \alpha}-g_{v \varrho} \varepsilon_{\gamma \sigma \alpha \tau \alpha}\right) \\
& \pm \frac{i}{2} \frac{X^{\alpha}}{S^{3}} \sigma_{\mathrm{F}}^{\alpha \dot{\mathrm{B}}} \sigma_{\dot{\mathrm{BA}}}^{\tau}\left(X_{v} X_{\varrho} \varepsilon_{\gamma \sigma \kappa \tau \alpha}+X_{\varrho} X_{\sigma} \varepsilon_{\gamma v \kappa \tau \alpha}-X_{\nu} X_{\sigma} \varepsilon_{\gamma \varrho \kappa \tau \alpha}\right) \mp i \varepsilon_{v \varrho \tau \sigma \alpha} \frac{X^{\alpha}}{S} \sigma^{\tau} \dot{\mathrm{DA}}_{\gamma} \sigma_{\gamma}^{\dot{\mathrm{D}}_{\mathrm{F}}} \text {. }
\end{aligned}
$$

Durch Verjüngung $F=A$ entsteht daraus die einfachere Formel:

$$
\begin{aligned}
& \sigma_{\nu} \dot{\mathrm{B}}_{\mathrm{A}} \sigma_{\varrho \dot{\mathrm{BC}}} \sigma_{\sigma \dot{\mathrm{D}}}{ }^{\mathrm{C}} \sigma_{\gamma}^{\dot{\mathrm{DA}}}=2\left[g_{\nu \varrho} g_{\gamma \sigma}+g_{\varrho \sigma} g_{\gamma \nu}-g_{\nu \sigma} g_{\gamma \varrho}\right]+\frac{2}{S^{2}}\left[g_{v \sigma} X_{\gamma} X_{\varrho}+g_{\gamma \varrho} X_{v} X_{\sigma}-g_{\varrho \sigma} X_{\gamma} X_{v}\right. \\
& \left.-g_{\gamma \nu} X_{\varrho} X_{\sigma}-g_{\nu \varrho} X_{\gamma} X_{\sigma}-g_{\gamma \sigma} X_{v} X_{\varrho}\right]+\frac{2}{S^{4}} X_{v} X_{\sigma} X_{\gamma} X_{\varrho} \pm 2 i \varepsilon_{v \varrho \gamma \sigma \alpha} X^{\alpha} / S .
\end{aligned}
$$

\section{$\S$ 2. Projektion des 5-dimensionalen $\sigma$-Apparates}

Für die Durchführung der Projektion ist die früher ${ }^{1}$ gefundene Verknüpfungsgleichung:

$$
\varepsilon_{i_{1} \ldots i_{4}}=\varepsilon_{i_{1} \ldots i_{\triangleleft} \alpha} X^{\alpha} / S
$$

von großer Wichtigkeit. Beachtet man noch, daß $\varepsilon_{m n r s i}=0$ wegen des Auftretens zweier gleicher Indizes sein muß, so resultiert durch Projektion von
(11), (12), (13) und (14) :

$\varepsilon_{n r t a} \varepsilon^{s l k b}$

$=\quad g_{n}^{s} g_{r}^{l} g_{t}^{b} g_{a}^{k}+g_{n}^{s} g_{r}^{k} g_{t}^{l} g_{a}^{b}+g_{n}^{s} g_{r}^{b} g_{t}^{k} g_{a}^{l}+g_{n}^{l} g_{r}^{s} g_{t}^{k} g_{a}^{b}$ $+g_{n}^{k} g_{r}^{s} g_{t}^{b} g_{a}^{l}+g_{n}^{b} g_{r}^{s} g_{t}^{l} g_{a}^{k}+g_{n}^{b} g_{r}^{k} g_{t}^{s} g_{a}^{l}+g_{n}^{k} g_{r}^{l} g_{t}^{s} g_{a}^{b}$ $+g_{n}^{l} g_{r}^{b} g_{t}^{s} g_{a}^{k}+g_{n}^{k} g_{r}^{b} g_{t}^{l} g_{a}^{s}+g_{n}^{b} g_{r}^{l} g_{t}^{k} g_{a}^{s}+g_{n}^{l} g_{r}^{k} g_{t}^{b} g_{a}^{s}$ $-g_{n}^{s} g_{r}^{l} g_{t}^{k} g_{a}^{b}-g_{n}^{s} g_{r}^{b} g_{t}^{l} g_{a}^{k}-g_{n}^{s} g_{r}^{k} g_{t}^{b} g_{a}^{l}-g_{n}^{l} g_{r}^{s} g_{t}^{b} g_{a}^{k}$ $-g_{n}^{b} g_{r}^{s} g_{t}^{k} g_{a}^{l}-g_{n}^{k} g_{r}^{s} g_{t}^{l} g_{a}^{b}-g_{n}^{k} g_{r}^{b} g_{t}^{s} g_{a}^{l}-g_{n}^{b} g_{r}^{l} g_{t}^{s} g_{a}^{k}$ $-g_{n}^{l} g_{r}^{k} g_{t}^{s} g_{a}^{b}-g_{n}^{b} g_{r}^{k} g_{t}^{l} g_{a}^{s}-g_{n}^{k} g_{r}^{l} g_{t}^{b} g_{a}^{s}-g_{n}^{l} g_{r}^{b} g_{t}^{k} g_{a}^{s}$, 


$$
\begin{aligned}
\varepsilon_{m n r t} \varepsilon^{s l b t}= & g_{m}^{b} g_{n}^{l} g_{r}^{s}+g_{m}^{l} g_{n}^{s} g_{r}^{b}+g_{m}^{s} g_{n}^{b} g_{r}^{l} \\
& -g_{m}^{s} g_{n}^{l} g_{r}^{b}-g_{m}^{b} g_{n}^{s} g_{r}^{l}-g_{m}^{l} g_{n}^{b} g_{r}^{s},
\end{aligned}
$$

$$
\begin{aligned}
& \varepsilon_{m n r t} \varepsilon^{m n l k}=2\left[g_{r}^{k} g_{t}^{l}-g_{r}^{l} g_{t}^{k}\right], \\
& \varepsilon_{m n r t} \varepsilon^{m n r k}=-6 g_{t}^{k} .
\end{aligned}
$$

In entsprechender Weise führen wir jetzt die Projektionen der Formeln (19), (20), (21), (22), (23), (24), (26), (28), (33), (34), (35) und (36) aus und erhalten beziehungsweise:

$$
\begin{aligned}
& \sigma_{m} \dot{\mathrm{B}}_{\mathrm{A}} \sigma_{n \dot{\mathrm{BC}}}=g_{m n} \gamma_{\mathrm{AC}} \pm \frac{i}{2} \varepsilon_{m n r t} \sigma_{\mathrm{A}^{r}} \sigma_{\mathrm{BC}}^{t} \text {, } \\
& \sigma_{m}{ }_{\mathrm{A}}^{\dot{\mathrm{B}}} \sigma_{n \dot{\mathrm{BC}}}+\sigma_{n}{ }_{\mathrm{A}}^{\dot{\mathrm{B}}} \sigma_{m \dot{\mathrm{BC}}}=2 g_{m n} \gamma_{\mathrm{AC}} \text {, } \\
& \sigma_{m}{ }_{\mathrm{A}}^{\dot{\mathrm{B}}} \sigma_{n \dot{\mathrm{BC}}}-\sigma_{n}{ }_{\mathrm{A}}^{\dot{\mathrm{B}}} \sigma_{m \dot{\mathrm{BC}}}= \pm i \varepsilon_{m n r t} \sigma^{r \dot{\mathrm{B}}}{ }_{\mathrm{A}} \sigma_{\dot{\mathrm{BC}}}^{t} \text {, } \\
& \sigma_{m}{ }_{\mathrm{A}}^{\dot{\mathrm{B}}} \sigma_{n \dot{\mathrm{BA}}}=-2 g_{m n}, \quad \sigma^{m \dot{\mathrm{BA}}} \sigma_{m \dot{\mathrm{BA}}}=-8, \quad \sigma^{m \dot{\mathrm{B}}}{ }_{\mathrm{A}} \sigma_{m \dot{\mathrm{BC}}}=4 \gamma_{\mathrm{AC}} \text {, } \\
& 6 \sigma_{\dot{\mathrm{DA}}}^{k}= \pm i \varepsilon^{k m n l} \sigma_{l \dot{\mathrm{D}}}{ }^{\mathrm{C}} \sigma_{n}{ }^{\dot{\mathrm{B}}} \sigma_{m \dot{\mathrm{BA}}}, \quad \sigma^{s} \dot{\mathrm{DC}} \sigma_{s \dot{\mathrm{BA}}}=2 \gamma_{\dot{\mathrm{D}} \dot{\mathrm{B}}} \gamma_{\mathrm{AC}}, \\
& \sigma_{r \dot{\mathrm{BC}}}\left(\sigma_{i}{ }_{\mathrm{A}}^{\dot{\mathrm{B}}} \sigma_{j \mathrm{\textrm {D }}}{ }^{\mathrm{C}}+\sigma_{j}{ }_{\mathrm{A}} \sigma_{i \dot{\mathrm{D}}} \dot{\mathrm{C}}^{\mathrm{C}}\right)=-2\left(\sigma_{j \dot{\mathrm{DA}}} g_{i r}+\sigma_{i \dot{\mathrm{DA}}} g_{j r}-\sigma_{r} \dot{\mathrm{DA}} g_{i j}\right), \\
& \sigma_{n}{ }_{\mathrm{A}}^{\dot{\mathrm{B}}} \sigma_{r \dot{\mathrm{BC}}} \sigma_{s} \dot{\mathrm{D}}^{\mathrm{C}}=g_{n s} \sigma_{r \dot{\mathrm{DA}}}-\dot{g}_{r s} \sigma_{n \dot{\mathrm{DA}}}-g_{n r} \sigma_{s \dot{\mathrm{DA}}} \pm i \varepsilon_{n r s t} \sigma_{\dot{\mathrm{DA}}} \text {, } \\
& \sigma_{n}{ }_{\mathrm{A}}^{\dot{\mathrm{B}}} \sigma_{r \dot{\mathrm{BC}}} \sigma_{s \dot{\mathrm{D}}}{ }^{\mathrm{C}} \sigma_{m} \dot{\mathrm{D}}_{\mathrm{F}}=\gamma_{\mathrm{FA}}\left(g_{n s} g_{m r}-g_{r s} g_{m n}-g_{n r} g_{m s}\right) \\
& \pm \frac{i}{2} \sigma_{\mathrm{F}}^{k \dot{\mathrm{B}}} \sigma_{\dot{\mathrm{BA}}}^{t}\left(g_{n s} \varepsilon_{m r k t}-g_{n r} \varepsilon_{m s k t}-g_{r s} \varepsilon_{m n k t}\right) \mp i \varepsilon_{n r s t} \sigma_{\dot{\mathrm{D} A}}^{t} \sigma_{m} \dot{\mathrm{D}}_{\mathrm{F}} \text {, } \\
& \sigma_{n}{ }_{\mathrm{A}}^{\dot{\mathrm{B}}} \sigma_{r \dot{\mathrm{BC}}} \sigma_{s \dot{\mathrm{D}}}{ }^{\mathrm{C}} \sigma_{m} \dot{\mathrm{D} A}=2\left(g_{n r} g_{m s}+g_{r s} g_{m n}-g_{n s} g_{m r}\right) \mp 2 i \varepsilon_{n r s m} \text {. }
\end{aligned}
$$

Durch Vergleich mit der bereits erwähnten Arbeit des Verf. ${ }^{4}$ ersieht man, daß der 4-dimensionale Formelapparat genau mit dem dort entwickelten übereinstimmt, weshalb wir unsere 5-dimensionale Axiomatik als die konsequente Verallgemeinerung der 4-dimensionalen anzusehen haben.

\section{NOTIZEN}

\section{Sichtbarmachung von Versetzungen auf Ober- flächen von Eisen-Nickel-Legierungen (50/50) durch chemisches Ätzen}

Von W. D. Hannibal

Institut für Werkstoffe der Elektrotechnik der Technischen Hochschule Aachen

(Z. Naturforschg. 15 a, 835-836 [1960] ; eingegangen am 30. Mai 1960)

Kürzlich ist über elektrolytische Ätzverfahren zur Sichtbarmachung von Korngrenzen, Kleinwinkelkorngrenzen und Versetzungen auf elektrolytisch polierten Oberflächen berichtet worden ${ }^{1}$. Chemische Ätzverfahren für Versetzungen auf Eisen-Nickel-Legierungen sind $\mathrm{m}$. W. bisher nicht bekannt geworden. Im folgenden werden einige Beobachtungen darüber mitgeteilt, wie das Ätzverhalten auf Eisen-Nickel-Legierungen (50/50) bei einer nur chemischen Atzung vom Eisen- und Nickelgehalt der Lösung und dem Oberflächenzustand abhängt.

Die Ätzung wird in geschlossenen Wägeschälchen bei Zimmertemperatur vorgenommen und dauert 12 bis 14 Stunden. Die Ätzlösung besteht
1. entweder aus ursprünglich reiner A2-Lösung ${ }^{2}$ oder aus A2-Lösung mit so geringer Eisen- und NickelIonenkonzentration, daß die Lösung nur ganz schwach gefärbt ist, oder

2. aus A2-Lösung, in der eine $\mathrm{Fe}^{++}$. und $\mathrm{Ni}^{++}$-Ionenkonzentration von etwa $0,05 \mathrm{Mol} / l$ durch anodische Auflösung von $50 / 50 \mathrm{Fe} / \mathrm{Ni}$ hergestellt wird. Eine störende $\mathrm{Fe}^{+++}$-Ionenkonzentration wird durch Bleche aus dem gleichen Material, die vor Benutzung der Ätzlösung einige Tage bei Raumtemperatur in ihr liegen bleiben, beseitigt. Spuren von Kupfer beeinträchtigen den Ätzvorgang nicht.

Die Proben werden vor dem chemischen Ätzen

a) mit A2 im Disa-Electropol ${ }^{3}$ oder mit ChromsäureEisessig nach MorRIs ${ }^{4}$ elektrolytisch poliert, oder

b) wie in a) elektrolytisch poliert und dann im gereinigten $\mathrm{H}_{2}$ bei irgendeiner Temperatur zwischen

1 W. D. Hannibal, Acta Met. 7, 697 [1959].

2 Ein Elektrolyt, der von der Firma P. F. D u j a r d in u. Co., Düsseldorf, geliefert wird.

3 Gebaut nach KnUth-Winterfeldt von H. Struers, Kopenhagen.

4 C. E. Morris, Metal Progr. 56, 696 [1949]. 